\title{
La révolution industrielle selon W.E. Rappard
}

\section{Victor Monnier}

\section{OpenEdition \\ Journals}

Édition électronique

URL : http://journals.openedition.org/ress/329

DOI : $10.4000 /$ ress.329

ISSN : 1663-4446

\section{Éditeur}

Librairie Droz

\section{Édition imprimée}

Date de publication : 1 août 2005

Pagination : 163-170

ISBN : 2-600-01027-0

ISSN : 0048-8046

Référence électronique

Victor Monnier, "La révolution industrielle selon W.E. Rappard », Revue européenne des sciences sociales [En ligne], XLIII-132 | 2005, mis en ligne le 30 octobre 2009, consulté le 01 mai 2019. URL : http://journals.openedition.org/ress/329; DOI : 10.4000/ress.329 
Victor MONNIER

\section{LA RÉVOLUTION INDUSTRIELLE SELON W. E. RAPPARD}

Pour bien comprendre la Révolution industrielle, il convient d'évoquer en quelques pages la personnalité exceptionnelle de son auteur, William Emmanuel Rappard (1883-1958), en retraçant d'abord ses années de formation, en rappelant ensuite les conditions dans lesquelles cet ouvrage a été élaboré et en mettant enfin en lumière les grandes étapes de sa carrière d'universitaire au service de son pays et de la communauté internationale.

\section{LES ANNÉES DE FORMATION (1883-1913)}

W. E. Rappard reçoit au cours de son enfance et de sa jeunesse une formation qui lui sera utile sa vie durant. Né à New York, le 22 avril 1883, il avait pour père Auguste Rappard (1851-1920), homme d'affaires d'origine thurgovienne spécialisé dans le commerce de la broderie de Suisse orientale et pour mère Julie Hoffmann (1860-1884) appartenant à la célèbre famille de rubaniers bâlois. Au cours des premières années de son existence, W. E. Rappard apprend sans efforts les langues qui lui seront fort précieuses: le français parlé avec son père, l'allemand et le suisse-alémanique dans lesquels il s'exprime avec la famille de sa mère, enfin l'anglais qu'il étudie dans ses premières classes aux Etats-Unis. Après quinze années passées dans le Nouveau monde, W. E. Rappard, qui possède la citoyenneté américaine, s'établit avec sa famille à Genève. Ce déménagement a une cause: le père de W. E. Rappard entend ramener son fils unique en Suisse avant qu'il ne soit devenu irrémédiablement américain.

Arrivé à Genève en 1899, W. E. Rappard entre au Collège de Genève et obtient en 1901 la maturité classique. Après des études à l'Université de Genève, il est licencié en droit en 1906. Avec ces distinctions académiques, il a également réussi son intégration dans la vie genevoise. Son indépendance d'esprit et sa spontanéité qui évoquent les mœurs américaines font de lui un ami recherché.

Les étapes de Berlin, Munich, Cambridge Mass., Paris et Vienne, de 1906 à 1913, sont-elles le fruit du hasard? Vraisemblablement, elles correspondent à son intérêt pour l'évolution des doctrines économiques qui vont devenir l'objet de ses études.

A l'Université Frédéric Guillaume de Berlin, W. E. Rappard est ébloui par les cours des professeurs Gustav Schmoller (1838-1917) sur les sciences politiques et

\footnotetext{
${ }^{1}$ Cette contribution devrait paraître prochainement en introduction à la réédition de W.E. Rappard, La révolution industrielle et les origines de la protection légale du travail en Suisse. Berne, Staempfli, 1914, VII+343 p.
} 
Adolf Wagner (1835-1917) sur les mécanismes de l'économie politique. Ces deux professeurs le familiarisent avec les préceptes de la jeune Ecole historique allemande dont ils sont les représentants attitrés. Leurs théories en économie remettent fondamentalement en question toutes les conclusions anciennes de l'économie politique. Comme il n'existe pas, selon eux, de véritables lois économiques à l'exemple de celle de la pesanteur, toute connaissance en ce domaine est relative. La mission de l'économiste est d'examiner, d'observer, de disséquer les phénomènes, en utilisant pour ce faire les matériaux d'investigation existants. Ainsi les économistes allemands de la jeune Ecole historique se concentrent-ils sur des problèmes pratiques: études de questions sociales, recherches historiques et descriptives. L'essentiel de ces travaux touche les institutions de l'antiquité et du moyen âge, les doctrines anciennes, l'histoire sociale, la statistique, l'organisation économique des nations modernes. Il en résulte ainsi un regain d'intérêt pour l'histoire et l'étude des institutions économiques.

A l'Université Louis Maximilien de Munich, W. E. Rappard suit les cours d'économie politique, de science des finances et des sciences économiques du professeur Lujo von Brentano (1844-1931). Ses leçons sont empreintes de rigueur, de discipline et de clarté. Brentano, l'excellent «historiste» de la jeune Ecole allemande, le pédagogue confirmé parachève ainsi la formation de W. E. Rappard.

Le séjour à l'Université Harvard donne à W. E. Rappard l'occasion de faire la connaissance des économistes américains qui, depuis 1870, sont en train de gagner une solide renommée internationale. Hostiles, pour la plupart à toute théorie, formés de manière empirique, ils ont obtenu leurs premiers succès en étudiant des problèmes concrets d'économie politique. Parmi eux, figure le professeur Frank William Taussig (1859-1940), dont A. Wagner a certainement suscité chez W. E. Rappard l'envie de suivre l'enseignement. Pour ce grand économiste, professeur d'économie à l'Université Harvard, l'élément concret primait et la spéculation n'intervenait que plus tard. Taussig avait d'ailleurs le don de combiner l'étude des faits avec l'analyse théorique. A l'époque où W. E. Rappard suit ses cours, Taussig est en train de prendre le virage qui l'amènera à l'analyse marginaliste. Il va de la sorte faire intervenir, dans la théorie des coûts comparatifs, relevant de la théorie du commerce international, la demande ou l'utilité, déterminée par les «goûts » des consommateurs, comme critérium de l'avantage de l'échange. Ce maître exigeant, qui n'appréciait que les étudiants doués et passionnés, s'intéresse à W. E. Rappard auquel il propose, comme sujet de thèse, l'étude des corporations d'affaires au Massachusetts.

A Paris, W. E. Rappard suit les cours de l'un des rares Français qui accepte la théorie de l'utilité marginale: Adolphe Landry (1874-1956), professeur d'histoire des doctrines économiques à l'Ecole pratique des hautes études de la Sorbonne. Favorable à la doctrine du socialisme, A. Landry était convaincu que les théories de Karl Marx (1818-1883) constituaient un acquis essentiel, mais estimait qu'elles devaient néanmoins être améliorées. Au capitalisme voué à l'échec par sa recherche incessante du profit, il fallait, selon lui, substituer un socialisme édifié sur l'expérience. Ses idéaux socialistes, il essayait de les adapter à l'analyse marginaliste, tout en prenant ses distances vis-à-vis des socialistes de stricte obédience marxiste, et de ceux qui voulaient apparenter le marginalisme au libéralisme. L'enseignement de Landry fit grande impression sur W. E. Rappard et exerça sur sa pensée politique une influence notable. 
Durant son séjour parisien, W. E. Rappard a comme souci principal d'achever sa thèse. Il la présente en 1908, à la faculté de droit de Genève. Dans l'introduction de cette étude: Les corporations d'affaires au Massachusetts ${ }^{2}$, il explique ce qui dans les corporations du Massachusetts l'a intéressé: l'examen structurel de ces institutions, la connaissance effective de leur fonctionnement et leurs relations avec l'histoire économique des Etats-Unis.

\begin{abstract}
Aucun traité de droit public ne saurait montrer le fonctionnement effectif des institutions démocratiques aussi clairement que l'étude historique du processus par lequel l'opinion publique s'affirme en lois, après s'être successivement manifestée par des brochures, des articles de journaux et des revues, des discours d'hommes politiques, des rapports de commissions parlementaires et des projets législatifs ${ }^{3}$.
\end{abstract}

Cet ouvrage, qui est avant tout une étude d'histoire du droit commercial avec une forte connotation d'histoire économique, donne ainsi la pleine mesure de l'influence de la jeune Ecole historique allemande sur son auteur.

Enfin, W. E. Rappard séjourne à Vienne où, comme auditeur libre à la faculté de droit, il fréquente les cours d'économie politique des professeurs Eugen Böhm, chevalier von Bawerk (1851-1914) et Eugen Philippovich von Phillippsberg (1858-1917), marginalistes de l'Ecole autrichienne. Les leçons de Carl Grünberg (1861-1940), spécialiste du socialisme et de la politique agricole de la monarchie danubienne, vont également l'influencer. C'est d'ailleurs ce professeur qui l'incitera à utiliser le matérialisme historique dans le domaine de la recherche, tout en remettant en cause certaines théories de K. Marx.

L'étape de Vienne est déterminante pour W. E. Rappard, car elle marque l'achèvement de sa formation d'économiste. Les connaissances qu'il a acquises dans cette matière vont lui être utiles sa vie durant; elles lui permettront, plus tard, d'apprécier la valeur des économistes et d'attirer les meilleurs à l'Institut universitaire de hautes études internationales qu'il dirigera à Genève comme Ludwig von Mises (1881-1973), Friderich August von Hayek (1899-1992). Par ailleurs, l'escale viennoise correspond à un point de départ. C'est là que W. E. Rappard prend conscience de sa vocation d'historien. Ayant travaillé sous la direction de C. Grünberg, qui l'avait incité à étudier la Suisse du XVIII ${ }^{\circ}$ et du $\mathrm{XIX}^{\mathrm{e}}$ siècle sous l'angle socio-économique, il a accumulé informations et sources qui lui seront précieuses pour la rédaction de ses deux premiers ouvrages majeurs: Le facteur économique dans l'avènement de la démocratie moderne en Suisse ${ }^{4}$ (1912) et La révolution industrielle et les origines de la protection légale du travail en Suisse (1914).

Les concours de circonstances sont parfois étonnants : en 1909, W. E. Rappard se trouve à Bâle parce que Eugen Philippovich le pousse à accepter un poste à l'Office international du Travail qui y siège. Après une première expérience dans l'univers de la protection des travailleurs, W. E. Rappard revient à Genève où il

${ }^{2}$ Rappard, W. E., Les corporations d'affaires au Massachusetts. Paris, V. Giard \& E. Brière, 1908, 279 p.

Ibid., p. 8.

${ }^{4}$ Rappard, W. E., Le facteur économique dans l'avènement de la démocratie moderne en Suisse. t. I. L'agriculture à la fin de l'ancien régime. Genève, Georg, 1912, 235p. 
assure durant un semestre la suppléance d'histoire économique à l'Université, puis il retourne à l'Université Harvard où, en 1912, il est nommé professeur assistant d'économie. A la rentrée universitaire de l'automne 1913, il est de retour à Genève, car désormais il sera professeur titulaire de la chaire d'histoire économique (1913-1928) puis de finances publiques (1915-1957) de son université.

\section{LA GENÈSE DE DEUX GEUVRES MAJEURES : LE FACTEUR ÉCONOMIQUE ET LA RÉVOLUTION INDUSTRIELLE}

Entre 1908 et 1911, W. E. Rappard récolte des matériaux considérables sur l'histoire économique et politique de la Suisse de 1750 à 1848. Cependant les recherches qu'il avait entreprises dès son stage à Vienne vont suivre une voie quelque peu différente de celle qu'il avait prévue. Initialement, il avait l'intention d'écrire une monographie sur le mouvement ouvrier et la révolution industrielle en Suisse. C'est finalement à l'agriculture sous l'ancien régime qu'il consacre sa première grande publication post universitaire. Le projet d'une vaste étude sur $L e$ facteur économique dans l'avènement de la démocratie moderne en Suisse est contrecarré par sa nomination en Amérique. Il doit se limiter à l'étude de la paysannerie à la fin du XVIII ${ }^{\mathrm{e}}$, époque préludant à l'avènement de la révolution industrielle; ce thème devait faire l'objet d'un second volume, dont W. E. Rappard envisageait ultérieurement la publication. Ce livre ne verra jamais le jour.

En abordant l'avènement de la démocratie moderne en Suisse sous son aspect socio-économique, W. E. Rappard fait ainsi œuvre d'historien, et qui plus est, œuvre originale; jusqu'à cette époque, en effet, l'historiographie suisse n'avait analysé celle-ci que sous l'angle institutionnel et politique. La vaste documentation contenue dans le facteur économique, bien maîtrisée et analysée, nous apprend comment la Suisse est parvenue au « seuil de l'ère démocratique ${ }^{5}$. Dans cet ouvrage, W. E. Rappard entend prouver que c'est l'évolution économique qui est déterminante; les transformations constitutionnelles n'en sont que les conséquences. En décrivant de manière précise ce qu'étaient les charges féodales à la veille de la révolution, il souligne le trait de génie des révolutionnaires qui alors détermina les paysans à embrasser leur cause: l'abolition de la dîme.

Une fois le facteur économique terminé, et pour mettre à profit la documentation amassée, W. E. Rappard se met alors à la rédaction d'une monographie sur la révolution industrielle en Suisse et sur les origines de la protection ouvrière. Achevée en 1914, elle a pour titre La révolution industrielle et les origines de la protection légale du travail en Suisse, et se voit décerner une subvention honorifique lors de l'Exposition nationale de Berne. Cette étude dont les origines remontent à son séjour viennois, selon Giovanni Busino (1932), «... a probablement été écrite alors que l'auteur sentait qu'il fallait absolument trouver une conclusion aux observations et aux remarques sur les bouleversements provoqués par l'introduction de l' industrie et partant connaître la dialectique du rapport entre capitalisme et démocratie» ${ }^{6}$.

${ }^{5}$ Busino, Giovanni, «Histoire et politique chez W. E. Rappard», [introduction à] W. E. Rappard, Economistes genevois au $19^{\circ}$ siècle. Genève, Droz, 1966, p. XI.

${ }^{6}$ Ibid., p. XIII. 
W. E. Rappard attache une grande importance au facteur économique dans l'évolution de la démocratie suisse. Il y a, par conséquent, comme il l'écrit «un rapport de causalité entre la révolution industrielle et l'élaboration de la législation ouvrière $»^{7}$. Et c'est dans la révolution industrielle qu'il va rechercher les racines de la législation ouvrière. «Pour comprendre une révolution, «relève-t-il, 《il faut en effet connaître la matière révolutionnée ${ }^{8}$, ce à quoi il se consacre dans la première partie. S'appuyant sur des sources très précises et une vaste documentation, il fait la démonstration que c'est dans les Cantons relativement démocratiques et à prépondérance protestante que s'est développée l'industrie, celle des matières textiles et celle de l'horlogerie principalement. Il en établit alors la répartition géographique et la distribution professionnelle. En seconde partie, il étudie la révolution industrielle en décrivant le passage de l'industrie artisanale à l'industrie moderne et en examinant les progrès techniques réalisés ainsi que les répercussions politiques qu'ils avaient entraînées. Enfin, dans une troisième partie, il retrace le développement du droit ouvrier en Suisse et fait à ce propos deux constatations intéressantes. La première est que le mouvement de protection légale du travail a été d'abord un mouvement suisse-alémanique; les Suisses romands y étaient défavorables, bien que convaincus de sa nécessité, en raison des inconvénients que cette législation allait inévitablement engendrer. La seconde est que la législation sociale en Suisse a été le fruit de la démocratie à la différence des autres Etats européens où elle fut élaborée par les pouvoirs publics sans le concours de ceux qui devaient en bénéficier.

Nous avons évoqué brièvement la formation intellectuelle de W. E. Rappard et l'influence qu'elle a exercée sur lui pour aboutir aux deux livres Le facteur économique et La révolution industrielle, considérés comme les premières études d'histoire économique de la Suisse. Ceux-ci ne sont que les premiers d'une bibliographie impressionnante qui compte plusieurs centaines de titres touchant au droit, particulièrement au droit constitutionnel, à l'histoire, à l'histoire économique, à l'économie, à la statistique et aux relations internationales. Parmi ces multiples ouvrages, qu'il nous soit permis de mentionner L'individu et l'Etat (1936) et La Constitution fédérale de la Suisse (1948) ouvrages de référence dans le domaine de l'histoire des institutions politiques de la Suisse.

Rappelons ici sa conception du travail de l'historien, qu'il évoque dans ce dernier ouvrage, sachant combien importante a été cette activité. «Ce que l'on est en droit de demander à un historien digne du nom de savant, c'est en fait l'instruction d'un procès beaucoup plus que son jugement. Et cette instruction même ne saurait être conduite avec trop de rigueur. C'est dire qu' elle doit s'inspirer de la seule volonté impartiale de connaître et de comprendre la réalité passée telle qu'elle a été, et non pas du désir tendancieux de suggérer, quant à ce passé ou même à l'avenir, des conclusions conformes aux vues et aux væux préconçus de l'auteur.»"

\footnotetext{
${ }^{7}$ Rappard, W. E., La révolution industrielle, op. cit., p. 313.

${ }^{8}$ Ibid., p. 10.

9 Rappard, W.E., La Constitution fédérale de la Suisse. Boudry, La Baconnière, 1948, p. 12.
} 
Il nous reste à mettre en lumière les principales étapes de sa carrière en expliquant comment ce jeune professeur d'une trentaine d'années va devenir célèbre en Suisse et à l'étranger.

\section{LES GRANDES ÉTAPES DE LA CARRIÈRE DE W. E. RAPPARD, UNIVERSITAIRE AU SERVICE DE SON PAYS ET DE LA COMMUNAUTÉ INTERNATIONALE}

W. E. Rappard se fait connaître dès 1917 , alors qu'il appartient à la mission suisse envoyée par le Gouvernement helvétique aux Etats-Unis pour défendre la position de la Suisse neutre et assurer son approvisionnement. Grâce à ses collègues de l'Université Harvard, il réussit à obtenir une entrevue personnelle avec Woodrow Wilson (1856-1924), le président des Etats-Unis et ancien professeur à l'Université de Princeton. Ce dernier se laisse aller à s'exprimer en toute liberté face à ce jeune collègue d'un petit pays. Il l'entretient avec une ardente conviction de son dessein de nouvel ordre mondial. W. E. Rappard l'écoute avec une grande attention: c'est l'avenir de l'Europe, et donc aussi de la Suisse, que le président laisse entrevoir à son hôte. En 1918, il a de nouveau l'occasion d'être reçu à la Maison blanche. Au cours de son tête à tête avec le président Wilson, il apprend que la Société des Nations doit naître de la paix et sa charte faire partie intégrante du traité qui mettra fin au conflit mondial. Ainsi, seules les nations ayant subi la guerre seront admises à la table des négociations; la Suisse neutre ne pourra donc pas participer à l'élaboration du Pacte de la Société des Nations. Cependant elle pourra y adhérer lorsque celle-ci sera constituée.

Les travaux de la Conférence de la paix débutent à Paris en janvier 1919. Ils ont pour objectif la création de la Société des Nations qui mettra un terme à l'état de guerre qui divise le monde d'alors. Afin de permettre à la Suisse d'être renseignée sur les débats qui s'y tiendront et de faire valoir son point de vue, les Américains conviennent avec le Conseil fédéral que W. E. Rappard sera l'envoyé officieux de la Confédération auprès d'eux. Il sera dès lors tenu informé de ce qui se passe au sein de la Conférence dans les différents domaines qui peuvent concerner la Suisse. Le rôle de W. E. Rappard sera essentiel, car ses démarches auprès des membres des délégations alliées vont concourir à la désignation de Genève comme siège de la Société des Nations ainsi qu'à l'entrée de la Suisse dans cette organisation avec son statut de neutralité.

En octobre 1920, Sir Eric Drummond (1876-1951) le secrétaire général de la Société des Nations demande à W. E. Rappard d'assumer la direction de la section des Mandats de cette société. Ces Mandats consistaient en une tutelle sur les anciennes colonies des empires vaincus lors de la guerre de 1914-1918, comme le Liban, la Palestine, l'Irak, le Sud-Ouest africain. Cette tutelle était exercée par des puissances mandataires telles la France, la Grande-Bretagne, la Belgique, l'Union Sud-Africaine pour le compte et sous le contrôle de la Société des Nations. W. E. Rappard répond alors qu'il y a beaucoup de choses dans le monde qu'il ignore, mais qu'il n'y en a pas qu'il ignore plus radicalement que les questions coloniales. «C'est justement, me répondit-il, pour cela que je fais appel à vous.» ${ }^{10} \mathrm{C}^{\prime} e s t$ ainsi

${ }^{10}$ William Emmanuel Rappard, «Le problème des colonies et des Mandats » in Arhiva pentru stiinta si reforma sociala (Bucarest) 1932, $\mathrm{n}^{\circ} 1-4$, p. 5. 
que W. E. Rappard sera le premier directeur de la section des Mandats, section qu'il devra organiser pour la rendre opérationnelle. Son passage aux Mandats qu'il quitte en 1924 fait de lui un expert des problèmes coloniaux, mais aussi un spécialiste de tous les domaines ayant trait à la Société des Nations.

W. E. Rappard revient alors pleinement à la vie académique genevoise tout en restant membre de plusieurs commissions importantes de la Société des Nations et de l'Organisation internationale du Travail. De 1926 à 1928, il est recteur de l'Université de Genève. Alors qu'il était encore directeur de la section des Mandats, l'idée lui était venue de créer un institut universitaire destiné à étudier les questions internationales. Il avait constaté combien les ressources des organisations internationales et la présence dans la Genève des Nations, de personnalités influentes pourraient être intéressantes et profitables aux étudiants avancés. Grâce à l'influence décisive du conseiller d'Etat André Oltramare (1884-1947) chef du département de l'instruction publique et à l'appui financier du Laura Spelmann Rockefeller Memorial, le projet se réalise et l'Institut universitaire de hautes études internationales ouvre ses portes en septembre 1927. Dès 1928 jusqu'en 1951, W. E. Rappard en assume la codirection avec le professeur Paul Mantoux (1877-1956) puis la direction jusqu'en 1955.

Tout au long des années trente, W. E. Rappard, qui est à nouveau recteur de l’Université de Genève de 1936 à 1938, dénonce la menace que représentent pour la paix tant le nationalisme politique que le nationalisme économique. Des intellectuels de grande stature, contraints à l'exil, vont trouver alors refuge à l'Institut universitaire de hautes études internationales tels Hans Kelsen (1881-1973), Gugliemo Ferrero (1871-1943), Ludwig von Mises, Wilhelm Röpke (1899-1966). L'Institut sera, durant cette époque bien sombre, une sorte de citadelle de la pensée libérale sur le continent européen.

Lorsqu'éclate la deuxième guerre mondiale, W. E. Rappard ne doute pas de l'entrée en guerre des Etats-Unis et de leur victoire finale. Face aux forces de l'Axe qui dès 1940 encerclent la Suisse, il est persuadé que la seule politique envisageable pour la Suisse est le silence, car, explique-t-il : «Le fait est que notre indépendance, notre intégrité comme aussi notre vie économique sont conditionnées par la bienveillance d'un régime étranger qui est aux antipodes du nôtre. Il est évidemment facile, dans l'intimité, de braver par ses propos le courroux d'un homme dont un geste peut, en quelques jours, effacer notre pays de la carte de l'Europe. Mais notre peuple le pardonnerait-il vraiment à son gouvernement s'il l'exposait à un tel danger en défendant avec virilité nos valeurs nationales? ${ }^{11}$ Convaincu de l'impossibilité de mener une action efficace sur la place publique, il rallie en septembre 1940 les rangs de l'Aktion nationaler Widerstand, association dont le but est de maintenir l'indépendance de la Suisse et en cas d'agression de résister à l'ennemi. Dans les circonstances du moment, les Suisses, estime-t-il, n'ont d'autre mission que «...d'atténuer, dans la mesure de leur force, les conséquences des cataclysmes qu'ils n'ont rien fait pour provoquer et qu'ils ne peuvent rien faire non plus pour arrêter.» ${ }^{12}$ Son action humanitaire, W. E. Rappard la

${ }^{11}$ Copie de la lettre de W. E. Rappard à H. L. Miéville du 4 septembre 1940 in Fonds W. E. Rappard déposés aux Archives fédérales (Berne), vol. 34 / 67.

${ }^{12}$ Copie de la lettre de W. E. Rappard à G. Hochreutiner du 23 juillet 1940 in Fonds W. E. Rappard déposés aux Archives fédérales (Berne), vol. 24 / 48. 
poursuit principalement au sein du Comité pour le placement des intellectuels réfugiés qu'il préside de 1942 à 1948. A nouveau, le Gouvernement suisse fait appel à lui en le désignant pour participer aux différentes négociations importantes avec les Alliés, qui ont lieu en 1942, 1945 et 1946 à Londres, Berne et Washington.

Dès la fin de la guerre, W. E. Rappard reprend du service auprès de l'Organisation internationale du Travail, comme membre de la Commission d'experts pour l'application des conventions et recommandations du travail, réactivée par le directeur ad interim du Bureau international du travail, Edward J. Phelan (1888-1967) et à laquelle il appartenait depuis 1927. Puis c'est en tant que délégué du gouvernement suisse qu'il participe à la Conférence internationale du Travail, et ce, jusqu'en 1957. Cette activité lui donne l'occasion de rester en relation constante avec les délégations alliées et d'informer le Conseil fédéral sur leur attitude à l'égard de la Suisse, principalement sur la question de l'entrée de celle-ci dans l'Organisation des Nations Unies. Sa grande réserve à l'égard de cette question s'explique par le fait que la Suisse ne pourrait adhérer sans renoncer à sa neutralité. En outre, les dispositions de la Charte prévues pour régir le Conseil de sécurité violent le principe d'égalité juridique reconnu à chaque nation, étant donné qu'elles contiennent des droits et obligations inégaux. Enfin les cinq grandes puissances se voient reconnaître implicitement la faculté de pratiquer une politique d'agression en toute impunité, par l'exercice du droit de veto. Dans l'hypothèse d'une invasion de l'Europe occidentale par l'Union soviétique, comme le redoute, W. E. Rappard, la future organisation serait totalement inefficace. Entre l'adhésion et le refus d'adhérer, reste la troisième voie qu'il recommande au Conseil fédéral: celle de l'étroite participation à toutes les activités techniques des Nations Unies. Au cours de ses multiples entrevues avec les Alliés, W. E. Rappard plaide le maintien à Genève des Organisations internationales, tâche dont il s'acquitte avec succès.

Cette brève évocation ne saurait nous faire oublier l'homme de relations publiques qu'a été W. E. Rappard. Tout au long de son existence, il a assuré la fonction combien importante de trait d'union entre les différentes cultures auxquelles il appartenait. Il a joué d'abord un rôle de liaison entre les Suisses euxmêmes, notamment par sa maîtrise parfaite du français et du suisse-allemand, puis avec l'étranger, spécialement avec le monde anglo-saxon dont il était également issu. Ce grand libéral, pragmatique, que son empirisme rendait hostile à tout dogmatisme et qui profita pleinement, sans jamais en abuser, de la totale liberté que lui conférait son statut d'universitaire, a joui d'une indéniable autorité tant à l'intérieur de son pays qu'à l'extérieur et en particulier au sein des organisations internationales établies à Genève.

Département d' histoire du droit et des doctrines juridiques et politiques Université de Genève 